\title{
Financement de la traction animale dans le contexte \\ de désengagement de l'Etat. Enseignements des cas du Nord- Cameroun, de l'Est Burkina Faso et du bassin arachidier du Sénégal
}

\author{
B. Wampfler ${ }^{1,2}$
}

\begin{abstract}
Mots-clés
Energie animale - Financement Crédit - Subvention - Association $d^{\prime}$ agriculteurs - Politique financière Agriculture - Cameroun - Burkina Faso - Sénégal.
\end{abstract}

\begin{abstract}
Résumé
La traction animale suppose un investissement important que les ménages agricoles les plus vulnérables peuvent difficilement réaliser sur fonds propres. Le recours au crédit est alors nécessaire. En Afrique de l'Ouest et centrale, les formes publiques de financement de la traction animale ont permis l'équipement de larges zones pratiquant les cultures de rente. Mais ces dispositifs publics s'avèrent impossible à pérenniser et sont aujourd'hui en très forte régression. Dans le vide laissé par l'Etat, émergent des innovations institutionnelles portées par les organisations paysannes et la microfinance. Ces deux secteurs expérimentent aujourd'hui à très petite échelle le financement de la traction animale mais rencontrent eux aussi des difficultés importantes: les ressources financières adaptées sont difficiles à mobiliser, les crédits à moyen terme sont difficiles à sécuriser, les financements fournis sont très largement insuffisants par rapport à l'ampleur de la demande. Quelques institutions, comme par exemple le réseau des Caisses d'épargne et de crédit agricole mutuel (Cecam) à Madagascar, expérimentent des financements innovants et prometteurs.
\end{abstract}

\section{INTRODUCTION}

Le contexte dans lequel se développe la traction animale en Afrique de l'Ouest et centrale se modifie profondément avec le désengagement des Etats et la libéralisation du secteur agricole. Avant la libéralisation, les services permettant l'accès et le développement de la traction animale (fourniture d'équipement et d'animaux dressés, crédit, intrants permettant l'intensification des systèmes de production et la rentabilisation de la traction animale, santé animale...) étaient principalement assurés par le secteur public, à travers les sociétés de développement, les projets, les banques publiques... Avec la libéralisation, ces services doivent se privatiser et s'insérer dans une logique économique : leur durabilité sera liée à leur capacité à répondre à la demande, à dégager une rentabilité, et à se structurer en institution financièrement et socialement viable. Les services financiers ont été parmi les premiers secteurs touchés par la libéralisation et consti-

1. Cnearc, 1101 avenue d'Agropolis, BP 5098, 34033 Montpellier Cedex 01 E-mail : betty.wampfler@cnearc.fr

2. Cirad, département Tera, 73 rue Jean-François Breton, 34398 Montpellier Cedex 5 tuent aujourd'hui un «laboratoire » particulièrement fertile pour comprendre les modes de reconstruction des services à l'agriculture dans un contexte de désengagement de l'Etat (9).

Le programme de recherche «La traction animale, composante essentielle des stratégies paysannes en Afrique de l'Ouest et centrale : quelles sont les pratiques de recherche face au désengagement des Etats ? » s'est attaché à étudier l'évolution des conditions de développement de la traction animale dans trois régions présentant des degrés différenciés de désengagement de l'Etat: la zone arachidière du Sénégal dont l'Etat s'est désengagé au début des années 1980, la zone Gourmanche du Burkina Faso encore faiblement encadrée par l'Etat, et le Nord-Cameroun où l'encadrement public est encore largement présent à travers une société cotonnière. La démarche de recherche adoptée repose sur une analyse systémique combinant l'étude des pratiques et stratégies paysannes avec une analyse des services d'appui à la traction animale (conseil, fabrication d'équipement, financement). Dans les trois zones étudiées, la problématique du financement de la traction animale se pose en des termes différents, en fonction du degré de désengagement de l'Etat et d'émergence d'organisations paysannes 
et d'institutions de microfinance. Dans une première partie, sont analysées les spécificités des besoins de financement de la traction animale ; la deuxième partie revient brièvement sur l'analyse des acquis et des limites du financement public de la traction animale ; la troisième partie analyse les perspectives de financement face au désengagement de l'Etat. En conclusion, nous essayerons de dégager des pistes d'actions et de recherche susceptibles d'améliorer le financement de la traction animale.

\section{- POURQUOI LA TRACTION ANIMALE EST-ELLE SI DIFFICILE A FINANCER?}

L'équipement de traction animale des exploitations comporte les animaux (bovins, équins, asins, camelins en milieu sahélien), le matériel aratoire (charrue, semoir, herse, sarcleuse...) et le harnachement ; la charrette et le tombereau peuvent compléter l'équipement de base. Le financement peut porter sur un équipement initial (NordCameroun et Gourma burkinabé) ou sur le renouvellement d'un parc d'équipement ancien et vétuste (bassin arachidier du Sénégal).

Le montant d'investissement requis varie en fonction du niveau d'équipement (mono- ou bi-attelage, type d'animaux, type et diversité de l'équipement), de la nature de l'équipement (industriel ou fabrication locale, neuf ou d'occasion) et du degré de disponibilité sur le marché local (en fonction du parc global de matériel, de la période de l'année...).

Dans les trois zones d'étude, l'investissement requis est le plus souvent élevé au regard des revenus monétaires annuels des ménages agricoles. La phase d'équipement peut être longue et difficile pour les plus démunis. L'accès à la traction animale mobilise l'épargne de plusieurs années ou requiert le recours au crédit. A défaut, cet accès peut se faire ponctuellement par la location ou l'échange mais il est alors tributaire des disponibilités locales d'attelage, pouvant entraîner des retards importants dans la mise en place des cultures et compromettre leur rentabilité. Les animaux représentent une part importante de l'investissement ; mais rares sont les systèmes financiers qui acceptent le risque que comporte le financement des animaux. Il y a donc dans la plupart des cas une mise de fonds importante à réaliser par le ménage. La capacité d'épargne des ménages dans les trois zones étudiées est limitée par la faiblesse des revenus monétaires annuels, conjuguée avec la pression économique et sociale forte qui s'exerce sur les chefs d'exploitation. Par ailleurs, les ménages continuant à marquer une préférence pour l'épargne en bétail traditionnelle, l'épargne monétaire reste difficile à mobiliser $(4,7)$.

S'il y a recours au crédit, celui-ci sera un crédit de moyen terme et de montant relativement important, deux facteurs qui conduisent à une prise de risque élevé pour le prêteur. Ce risque est renforcé encore quand il s'agit de financer les animaux, qui peuvent disparaître ou perdre de la valeur par manque de soins par exemple. Pour couvrir ce risque, le prêteur aura recours à une demande de garantie. La capacité des ménages agricoles non équipés à fournir cette garantie varie en fonction de leur patrimoine mais est le plus souvent faible et handicapée par le contexte institutionnel et juridique (absence de titre foncier par exemple).

Même si la traction animale permet d'améliorer les performances de l'agriculture vivrière, les trois études de cas montrent que son développement est le plus souvent lié à l'adoption d'une culture de rente permettant de dégager des revenus monétaires (arachide au Sénégal, coton au Burkina Faso et Cameroun) sûrs et réguliers. Le financement est facilité si l'équipement de traction animale génère une rentabilité monétaire directe : ainsi observe-t-on un développement rapide de l'équipement en charrettes dans les zones où il existe un marché du transport actif ; ce développement est beaucoup plus lent dans les zones où la demande de services de transport est faible et ne permet pas de rentabiliser une charrette (zone de Botou dans le Gourma burkinabé par exemple). Le même processus est observé pour le semoir et les houes mécaniques au Sénégal, et la charrue en zone cotonnière (8).

La rentabilité de la traction animale est conditionnée par la conjugaison de différents facteurs endogènes et exogènes à l'exploitation: disponibilité foncière, disponibilité de main d'œuvre, capacité de fertilisation, changement de pratiques pour limiter l'érosion... Cette rentabilité peut être différée dans le temps, l'acquisition d'une technique sure demandant un apprentissage. Audelà des contraintes inhérentes à l'exploitation et au contexte agricole, la rentabilité de la traction animale dépendra de la qualité des services d'appui à l'agriculture, mais aussi de la qualité de l'accès au marché (pour l'accès aux animaux, le renouvellement des pièces de l'équipement, pour la commercialisation des productions qui permettront de rentabiliser la traction animale). Le financement de la traction animale peut être facilité dans les contextes ruraux où la diversification des activités économiques des ménages permet de générer des revenus monétaires complémentaires - voire plus importants - que les activités agricoles elles-mêmes.

Les besoins de financement de la traction animale s'inscrivent dans un ensemble de besoins de l'exploitation agricole (besoins de financement de campagne agricole, de stockage), mais aussi plus largement de l'unité ménage/famille : besoins de financement d'activités productives non agricoles, mais aussi besoins de consommation (vivres, écolage...) et parfois besoins de financement d'urgence. C'est donc en fonction de cet ensemble de besoins que devra être raisonné le financement de la traction animale (6).

Les besoins de financement s'expriment au niveau des exploitations agricoles, mais aussi au niveau des artisans qui, dans un contexte libéralisé, peuvent prendre le relais de l'industrie pour la fabrication du matériel. Un frein important au développement d'un artisanat de proximité est le manque de fonds de roulement permettant l'achat de la matière première et des pièces importées qui composent certains équipements aratoires. Les études menées dans les trois pays montrent que les ateliers sont en général de très petite taille, faiblement dotés en patrimoine susceptible de constituer une garantie pour un emprunt. Les ateliers sont le plus souvent dépourvus de comptabilité et leur gestion budgétaire n'est pas dissociée de celle du ménage. Les comptes d'exploitation et la rentabilité de l'activité sont de ce fait difficiles à appréhender par un éventuel prêteur. Par ailleurs, le secteur artisanal est faiblement organisé et a peu de contacts avec les systèmes de financement de proximité et encore moins avec les banques. Au Sénégal et au Burkina Faso, des projets ont appuyé le secteur artisanal (fabrication de matériel, appui à la gestion, accompagnement de l'organisation du secteur) mais touchent davantage l'artisanat urbain que l'artisanat rural.

\section{- FINANCEMENT PUBLIC DE LA TRACTION ANIMALE : UN BILAN MITIGE}

Le financement public de l'agriculture repose sur l'hypothèse du cercle vertueux de l'investissement : l'injection de crédit dans les économies rurales permettrait d'amorcer une augmentation des revenus qui à son tour engendrerait l'investissement privé. Dans cette optique, le développement de la traction animale a été financé pour une large part par des fonds publics sous forme de crédit, conjugués avec des subventions. Trois types d'institutions ont été utilisés pour «acheminer » ce financement vers les bénéficiaires : les banques publiques, associées aux sociétés de développement, et les projets de développement. 
Ces trois dispositifs de financement ont plusieurs points communs. Le crédit pour la traction animale est mis en ouvre dans le cadre du développement d'une culture de rente (arachide, coton...). Le plus souvent, cette culture est l'unique source de revenus monétaires des ménages et doit permettre de faire face aux différents emprunts agricoles : intrants, équipement, crédits sociaux. Les taux d'intérêt pratiqués sont bas, subventionnés, pour favoriser l'investissement rural. L'accès au crédit n'est pas lié à l'épargne et la fonction d'épargne est peu développée, même par les banques. Le dispositif le mieux sécurisé est celui des sociétés de développement, le remboursement du crédit étant assuré par un prélèvement direct sur la récolte dont la société de développement détient le monopole de collecte et de commercialisation. Dans les deux autres dispositifs, banques et projets de développement, la sécurisation du crédit est plus aléatoire et repose sur le suivi technique et diverses formes de caution solidaire par les groupements de producteurs. Dans les trois cas, le crédit s'insère dans un ensemble de services organisés autour de la culture de rente (accompagnement technique, approvisionnement, vulgarisation, commercialisation...) qui contribuent à en renforcer la sécurisation et la rentabilité.

Le bilan de trois décennies de financement public de la traction animale en Afrique de l'Ouest et centrale est mitigé. De vastes régions de cultures de rente (zones cotonnières, zones arachidières) ont été équipées en traction animale (encadré 1). En revanche, de nombreuses régions d'agriculture vivrière ont été exclues de ces financements. La région du Gourma au Burkina Faso en est un exemple (1). Par ailleurs, le financement public de la traction animale s'est avéré difficile à pérenniser. Les taux d'intérêts faibles, inférieurs au coût réel du crédit, n'ont pas permis de couvrir les frais de fonctionnement et de reproduction du système de crédit.

Les systèmes de garantie défaillants et l'absence de recours juridique ont limité les possibilités de pression coercitive. Les faibles taux de remboursement des crédits bancaires et des crédits des projets, conjugués souvent à une gestion laxiste, ont renforcé les déficits des structures de crédit. Les banques agricoles se sont avérées être des structures coûteuses, difficiles à gérer et à décentraliser. Le crédit des banques comme celui des projets a été souvent utilisé à des fins politiques, entraînant une détérioration profonde et durable des mentalités par rapport au crédit.

Tant que les filières de cultures de rente sont restées fortement intégrées, les systèmes de crédit des sociétés de développement ont globalement mieux résisté. Mais la sécurisation par le prélèvement direct sur la collecte de culture de rente montre également des limites. Des baisses de production conjuguées à la défaillance

\section{Encadré 1}

\section{LA TRACTION ANIMALE DANS LE BASSIN ARACHIDIER DU SÉNÉGAL}

En 1980, à l'issue de la période active de financement public de l'équipement, le parc de matériel de traction animale était évalué à 190000 semoirs, 296000 houes, 124000 charrettes, 82000 souleveuses, 56000 charrues, 8900 butteurs et 2000 unités de culture attelée. Pendant les vingt années suivantes, dans un contexte de désengagement complet et brutal de l'Etat, très peu de matériel neuf a été acquis dans le bassin arachidier. Le parc initial est maintenant vétuste et malgré I'habilité des artisans à le réparer, demande à être renouvelé.

Source : Havard et Wampfler, 2003, Cirad des systèmes de caution solidaire au sein des organisations paysannes intermédiaires ont conduit ponctuellement à des situations d'endettement des producteurs et de blocage des systèmes de crédit. Là encore, l'utilisation politique du crédit, qui a souvent conduit à l'effacement des dettes, a pu favoriser la gestion laxiste et la détérioration des mentalités par rapport au crédit (5).

Avec le recul de l'aide internationale, les ressources publiques dévolues à ces financements ont fortement baissé. Comme aucun de ces systèmes de financement n'était appuyé sur l'épargne, l'épuisement de la ressource financière a mis en évidence les faibles perspectives de pérennisation de ces systèmes de financement. Au fil des années 1980, dans le contexte de libéralisation croissante, les échecs sévères de ces modes de financement publics et la difficulté de les pérenniser ont conduit à en faire brutalement table rase (comme dans le cas de la nouvelle politique agricole du Sénégal) ou à les réduire plus progressivement (Burkina Faso).

\section{QUELLES PERSPECTIVES DE FINANCEMENT DE LA TRACTION ANIMALE FACE AU DESENGAGEMENT DE L'ETAT?}

Les trois études de cas montrent que l'offre de financement de la traction animale a été drastiquement réduite au cours de la dernière décennie. Même s'il reste des contributions publiques, l'offre repose aujourd'hui essentiellement sur les innovations institutionnelles constituées par l'action collective et les institutions de microfinance.

La libéralisation n'a pour l'instant pas abouti à la structuration d'une offre de financement de la traction animale par le secteur privé. Les banques commerciales classiques sont très peu présentes dans le financement du monde rural et inexistantes dans le financement de la traction animale. Les enquêtes n'ont pas permis d'identifier un crédit fournisseur d'envergure. Tout juste peut-on observer ponctuellement le développement de pratiques informelles (épargne progressivement déposée par un acheteur auprès d'un artisan forgeron...).

\section{Que reste-t-il du financement public de la traction animale?}

\section{L'offre de financement de la traction animale par les banques régresse}

Le Nord-Cameroun ne compte aucune banque publique finançant la traction animale. Les banques agricoles qui restent actives au Burkina Faso et au Sénégal conservent un portefeuille encore fortement rural, relativement diversifié pour la Caisse nationale de crédit agricole (Cnca) du Sénégal, fortement concentré sur la filière coton pour la Banque agricole et de commerce du Burkina Faso (nouvelle dénomination de la $\mathrm{Cnca}$ ). Toutes deux proposent un crédit moyen terme pour la traction animale. Mais les volumes de crédit engagés sont faibles au regard des besoins et ont fortement régressé dans les années récentes (tableau I).

Ces évolutions s'expliquent à la fois par les difficultés de recouvrement des crédits (impayés importants dans les filières cotonnières) et par les évolutions du contexte économique et institutionnel (crise des agricultures familiales, désorganisation de filières porteuses, difficulté de décentralisation des banques) (14).

Certaines orientations stratégiques peuvent influer sur l'offre de financement de la traction animale par les banques agricoles. L'alliance avec les organisations paysannes se développe et vise notamment à faciliter l'identification des besoins et l'organisation du financement (l'Union nationale des producteurs de coton du 
Tableau I

Financement de la traction animale par les banques publiques du Burkina Faso et du Sénégal

\begin{tabular}{|c|c|c|}
\hline & $\begin{array}{c}\text { Bacb Burkina } \\
\text { Crédit traction } \\
\text { animale pour trois } \\
\text { provinces de l'Est } \\
\text { (millions Fcfa) }{ }^{*}\end{array}$ & $\begin{array}{l}\text { Cnca Sénégal } \\
\text { Crédit matériel } \\
\text { agricole } \\
\text { Echelle nationale } \\
\text { (millions Fcfa) }{ }^{* *}\end{array}$ \\
\hline 1997-1998 & 100 & 1017 \\
\hline 1998-1999 & 56,5 & 1248 \\
\hline $1999-2000$ & 24,5 & 1284 \\
\hline $2000-2001$ & Moins de 20 & 586 \\
\hline 2001-2002 & 16 (sur une ligne de 25) & 554 \\
\hline $2002-2003$ & 25 mis à disposition & Prévision 109 \\
\hline
\end{tabular}

Bacb : Banque agricole et de commerce du Burkina Faso

Cnca : Caisse nationale de crédit agricole

* Communication de la Bacb à l'atelier ATP Fada, septembre 2002

** Communication Cnca du Sénégal à la mission Havard et Wampfler, février 2003, Cirad

Burkina Faso [Unpcb] en est un exemple). L'alliance des banques avec la microfinance offre une alternative face aux difficultés de décentralisation, de suivi et de recouvrement des crédits par les banques agricoles. Même si les résultats sont pour l'instant mitigés, ce partenariat continue a être expérimenté par les banques agricoles du Burkina Faso et du Sénégal sous différentes formes (refinancement, formation professionnelle, participation à la création de petites mutuelles...). En revanche, l'aspiration commune des banques agricoles à devenir "banques universelles », élargissant leur clientèle au-delà du rural, risque de limiter les investissements dans l'équipement agricole qui reste coûteux et risqué.

\section{L'offre de financement de la traction animale des sociétés de développement diminue}

Parmi les trois sociétés cotonnières encore en exercice, seule la Société de développement du coton (Sodecoton) du Nord-Cameroun fait du crédit direct à ses producteurs. La Société des fibres textiles (Sofitex) du Burkina Faso et la Société de développement des fibres textiles (Sodefitex) du Sénégal gèrent le recouvrement des crédits traction animale consentis par les banques agricoles. Mais leur rôle reste néanmoins déterminant en matière de financement de la traction animale : elles structurent le développement de la filière agricole qui fournit la majeure partie des ressources financières que les exploitations agricoles peuvent investir dans la traction animale ; en détenant le monopole de la collecte du coton, elles restent un outil de sécurisation du crédit des banques agricoles. Mais les résultats sont là aussi mitigés.

Le système de crédit traction animale de la Sodecoton repose sur les groupes de caution solidaire constitués par les groupements de producteurs de coton. Le crédit porte uniquement sur l'équipement, les animaux ne sont pas financés. Il est consenti sur deux ans, à un taux d'intérêt de 10 p. 100 par an, moyennant un apport personnel de 25 à 30 p. 100. Le système permet de financer de 3000 à 4000 charrues par an pour un montant annuel de 400 à 700 millions Fcfa $(3,6)$.

Au Sénégal, le programme Caisse nationale de crédit agricole du Sénégal (Cncas)/Sodefitex de financement de matériels agricoles a connu des difficultés importantes (tableau II).

\section{Tableau II}

Evolution des financements de matériel agricole du programme Cncas/Sodefitex Sénégal (1995-2001)

\begin{tabular}{lcccc}
$\begin{array}{l}\text { Campagnes } \\
\text { agricoles }\end{array}$ & $\begin{array}{c}\text { Montants } \\
\text { crédits } \\
\text { matériel } \\
\text { agricole } *\end{array}$ & $\begin{array}{c}\text { Nb. de } \\
\text { bénéfi- } \\
\text { ciaires }\end{array}$ & $\begin{array}{c}\text { Taux de } \\
\text { rembour- } \\
\text { sement ** } \\
(\%)\end{array}$ & $\begin{array}{c}\text { Volume } \\
\text { des } \\
\text { impayés * }\end{array}$ \\
\hline $1995-96$ & 80,8 & 35 & 75 & 26,6 \\
$1996-97$ & 12,3 & 12 & 50 & 7,9 \\
$1997-98$ & 75,8 & 16 & 33 & 45,3 \\
$1998-99$ & 93,6 & 19 & 0 & 55,9 \\
$1999-2000$ & 73,6 & 13 & 82 & 3,3 \\
$2000-01$ & 50,9 & 9 & $n d$ & $n d$ \\
Total & 387,2 & 104 & 47 & 139
\end{tabular}

Source : Cncas zone Sud

Cncas : Caisse nationale de crédit agricole du Sénégal ; Sodefitex : Société des fibres textiles

* Millions de Fcfa

** Au 02/2003

nd : non déterminé

Dans la région Est du Burkina Faso, la Banque agricole et de commerce du Burkina Faso (Bacb) indique qu'environ 70 p. 100 des impayés des crédits moyens terme proviennent de crédits à la traction animale (communication Bacb, atelier ATP Fada N'Gourma, septembre 2002).

Les défaillances du système de sécurisation du crédit et les perspectives des filières cotonnières peuvent, à brève échéance, compromettre ces dispositifs de financement public déjà fortement ébranlés.

\section{Une offre réduite de financement de la traction animale} subsiste au sein de projets et programmes de développement

Au Burkina Faso, la région Est a bénéficié de deux programmes nationaux de promotion de la culture attelée mis en œuvre au niveau national à partir de 1991 : l'opération «30 000 charrues », financée par la coopération autrichienne, visait à accélérer le rythme de la mécanisation et l'augmentation de la productivité agricole (1991-1993), et l'opération « 2000 multiculteurs », engagée par l'Etat dans le prolongement de la précédente opération (1996-1997). A l'échelle nationale, ces deux opérations, complétées par trois autres programmes qui n'ont pas été appliqués dans l'Est - le Fonds de l'eau et de l'équipement rural (Feer), l'Unama, le Programme d'appui a la mécanisation agricole (Pama) - ont financé plus de 70000 matériels de traction animale pendant la décennie 1990 (1). Dans les deux programmes, le matériel de traction animale était octroyé à crédit à des groupements (villageois, groupements de jeunes, centres de formation...) chargés d'assurer la redistribution au producteur, et la collecte des remboursements de crédit, sous le contrôle de l'administration. Les crédits étaient octroyés à un taux nul, sur une durée de cinq à sept ans. Les taux de remboursement des crédits ont été extrêmement faibles (moins de 20 p. 100) dans chacune de ces opérations au niveau national.

Au Burkina Faso toujours, le Pama a initié un partenariat avec une organisation paysanne, l'association Tin Tua, pour la mise en œuvre d'un programme expérimental d'appui à la gestion du passage de la culture manuelle à la culture attelée, comportant une ligne de crédit pour la traction animale. Les acquis et les limites de cette expérience illustrent la difficulté des organisations paysannes à gérer le financement de l'équipement agricole (14). 
Au Sénégal, malgré le désengagement précoce de l'Etat, il subsiste des programmes d'appui à la modernisation de secteurs économiques en difficulté. Deux d'entre eux touchent à la question du financement de l'équipement agricole, le Programme de modernisation et d'intensification de l'agriculture (Pmia) et le projet de Promotion des micro-entreprises rurales (Promer). Par rapport aux projets antérieurs, le mode d'intervention de ces programmes a évolué. Leur action est centrée sur la consolidation institutionnelle des secteurs économiques (appui aux organisations et aux entrepreneurs de l'agriculture et de l'artisanat). Les fonds de crédit dont ils disposent doivent être octroyés par des voies pérennes et sont de ce fait majoritairement confiés aux institutions de microfinance.

\section{Périphériques au financement, mais vitaux pour sa sécurisation, les services publics d'accompagnement de la traction animale se désagrègent}

Les services publics d'accompagnement de la traction animale contribuaient fortement à la sécurisation et la rentabilisation de son financement. Ces services sont aujourd'hui intacts dans la zone cotonnière du Nord-Cameroun, très fortement dégradés dans l'est du Gourma, et dégradés mais en recomposition sous une forme «privatisée » au Sénégal, à travers l'expérience de l'Agence nationale du conseil agricole et rural (Ancar). La dégradation des services d'accompagnement a un impact négatif direct sur la diffusion de la traction animale, mais aussi plus globalement sur les conditions de sa rentabilisation au sein des exploitations. Ainsi, au Sénégal par exemple, la rupture de l'approvisionnement en semences d'arachide, les approvisionnements sporadiques en intrants et l'absence de conseil agricole ont contribué à la baisse drastique de la production arachidière qui compromet les capacités d'autofinancement des exploitations.

\section{Dans le vide laissé par l'Etat, émergent des innovations institutionnelles au sein des organisations paysannes et de la microfinance}

Financement de la traction animale : une préoccupation des organisations paysannes

La libéralisation et la décentralisation ont stimulé l'émergence des organisations paysannes dans la dernière décennie. Leur degré de structuration est extrêmement variable selon les contextes : elles sont faiblement développées et limitées au secteur coton au NordCameroun, embryonnaires dans l'Est Burkina Faso et plus fortement développées au Sénégal. Le financement de l'équipement agricole est une préoccupation centrale pour la plupart de ces organisations. Face à la baisse drastique des financements publics et à l'insuffisance des apports des systèmes de financement décentralisés, les organisations paysannes expérimentent quatre principales formes de réponse (10).

Dans un premier temps, elles sont souvent tentées de mettre en place un service de crédit interne à l'organisation. Fondé sur une subvention ou une ligne de crédit confiée par un bailleur de fonds, éventuellement complétée par des ressources propres, le crédit est géré par l'organisation paysanne qui en assure la conception, l'octroi et le recouvrement. L'expérience de l'association Tin Tua (Est Burkina Faso) est illustrative des grandes difficultés rencontrées par ce type d'initiative (8). Les taux de remboursement obtenus sont en général faibles. Les taux d'intérêt pratiqués ne permettent pas la pérennisation du service. Les outils et les compétences de gestion financière de l'organisation paysanne sont insuffisants. L'offre de crédit de moyen terme est limitée, notamment faute de ressources disponibles à moyen terme. La pérennité des services ainsi créés est très incertaine. L'offre de financement est souvent limitée dans le temps, ce qui favorise les comportements opportunistes des emprunteurs. La difficulté est renforcée dans le cas des crédits de moyen terme à la traction animale, dont les montants en jeu sont plus élevés et les durées de prise de risque plus longues.

Une autre voie consiste pour l'organisation paysanne à créer une structure de financement autonome, le plus souvent d'inspiration mutualiste. Le Sénégal offre l'exemple d'organisations paysannes qui ont créé des structures de financement ayant aujourd'hui une portée significative et capables, dans une certaine mesure, de prendre en compte le financement de l'équipement productif (dans le secteur de la pêche notamment, avec les mutuelles de la Fenagipêche). Tout en étant plus viable, cette option se heurte alors aux problèmes classiques rencontrés par la microfinance pour financer l'équipement agricole.

Créer un partenariat avec des institutions financières existantes est une troisième voie expérimentée par des organisations paysannes dans les zones où ces institutions existent. Ce type de partenariat s'avère cependant difficile à établir entre deux secteurs qui se connaissent peu et portent souvent le poids d'une histoire conflictuelle. Là encore, le partenariat, s'il est établi, le sera prioritairement pour le crédit de court terme et ne portera, au moins dans un premier temps, que très marginalement sur le crédit moyen terme nécessaire pour la traction animale.

Enfin, influer sur le cadre macroéconomique et institutionnel est une autre contribution possible des organisations paysannes à la question du financement agricole. Au Sénégal par exemple, les organisations paysannes ont ainsi obtenu la participation au capital de la Cnca, la création d'outils de bonification du crédit à l'équipement agricole et de sécurisation du financement agricole (fonds de garantie). Ces outils ont été mis en place par l'Etat sénégalais entre 1997 et 1999, puis ont été remis en cause par manque de ressources. Autre exemple de la mobilisation des organisations paysannes pour la définition de politiques et de dispositifs de financement de l'agriculture : en 2004, l'Etat burkinabé a confié à l'Unpcb des actions prioritaires dans le cadre stratégique de développement de la filière coton, portant notamment sur l'identification des besoins de financement d'équipement agricole, et l'intermédiation entre les producteurs et les institutions financières pour l'organisation du financement.

\section{Offre de financement de la traction animale par la microfinance}

La microfinance a émergé dans les années 1980, dans un contexte de libéralisation, en réponse à l'échec des formes et modes de financement antérieurs par les banques agricoles publiques, les banques de développement et les projets de développement. L'émergence de ce secteur correspond à un changement conceptuel dans l'approche du financement rural. L'objectif n'est plus d'injecter du crédit dans une production agricole, mais de construire un marché financier rural qui permette un accès durable aux services financiers, en mettant en relation les agents disposant de ressources monétaires (épargnants) avec ceux qui en ont besoin (les emprunteurs). Il ne s'agit plus de financer une production ou une technique agricole donnée mais de promouvoir des activités économiques diversifiées et de donner aux populations exclues du système bancaire les moyens de développer des activités génératrices de revenus et permettant l'accumulation. Pour que ces marchés financiers ruraux puissent fonctionner durablement, les institutions de microfinance recherchent l'autonomie et la pérennité. Le taux d'intérêt payé par l'emprunteur doit permettre de couvrir le coût des services financiers et d'assurer la reproduction de l'institution. Les innovations en matière de garantie (caution solidaire) et d'organisation (appropriation et gestion par les bénéficiaires) visent à ouvrir l'accès aux services financiers aux populations défavorisées et à assurer la viabilité sociale des institutions de microfinance. 
Dans les huit pays de l'Union monétaire et économique des Etats de l'Afrique de l'Ouest (Umeoa), on recensait en fin 2000 environ 3000 points d'accès de microfinance bénéficiant à 4,3 millions de personnes. Ces institutions mobilisaient à cette date 116,8 milliards Fcfa d'épargne et avaient un encours de 103,4 milliards Fcfa de crédit. En Afrique centrale, le développement de la microfinance est plus récent et plus difficile à appréhender faute de système d'information adéquat : en 2000, 1034 structures de microfinance étaient répertoriées dans les pays de la Communauté économique et monétaire de l'Afrique centrale (Cemac), touchant 411000 membres. Même si les volumes financiers traités par les institutions de microfinance restent marginaux par rapport au secteur bancaire, leur forte décentralisation constitue un avantage comparatif indéniable.

Malgré une forte proximité avec le monde rural, on observe que la microfinance répond avec difficulté aux besoins spécifiques du financement des agricultures familiales. Les institutions de microfinance rurales financent spontanément le développement d'activités rurales telles que le commerce, l'artisanat, la transformation agroalimentaire. Ces activités génèrent des revenus réguliers, relativement sûrs, avec des cycles de rotation du capital rapides, limitant les risques et permettant des taux de rentabilité élevés. Les activités agricoles présentent, au contraire, des degrés de risque importants, et une rentabilité souvent aléatoire ; elles requièrent du crédit de moyen terme que la microfinance assure avec difficulté. Ces facteurs contribuent à expliquer la grande prudence que montre la plupart des institutions de microfinance à l'égard du crédit agricole. L'objectif de durabilité des services financiers renforce cette tendance, les institutions de microfinance étant naturellement portées à investir dans les secteurs économiques les plus rentables et les moins risqués pour sécuriser leur pérennisation (12).

La microfinance est présente dans les trois régions étudiées à des stades de développement différenciés. Le crédit octroyé est essentiellement de court terme, mais on observe quelques expérimentations de crédit de moyen terme permettant de financer l'équipement agricole (Rpso, Association pour le développement de la région de Kaya [Adrk], Union des coopératives d'épargne et de crédit du Zoundwéogo [Ucec-Z] au Burkina Faso et Crédit mutuel du Sénégal $[\mathrm{Cms}])$. Hormis l'Adrk, dont la mutuelle finance à grande échelle la traction animale, les autres expériences restent plus marginales.

En l'état actuel de son développement, financer la traction animale reste difficile pour la microfinance. Sa fragilité qui demeure grande, limite sa capacité à prendre des risques et à innover. Souvent, elle connait mal l'agriculture et ne dispose pas de référentiels technico-économiques adaptés pour juger de la qualité de dossiers de crédit agricole. La rentabilité à court terme de la traction animale est souvent difficile à établir et demande une analyse globale de l'économie de l'exploitation ou du ménage que la microfinance n'est pas aujourd'hui en mesure de faire. Par ailleurs, des décennies de crédits équipement mal remboursés ont donné à la traction animale une mauvaise réputation. La traction animale requiert des volumes de financement importants alors que la microfinance ne dispose que de ressources limitées, mobilisées en priorité pour satisfaire la demande de crédit de court terme. Le financement de la traction animale est risqué et les formes de garantie innovantes promues par la microfinance, fondées sur le principe de la caution solidaire, s'adaptent mal au crédit moyen terme. La microfinance a des taux d'intérêt élevés, justifiés par la nature et la structure du service rendu (gestion d'un grand nombre de petits crédits, éloignement et dispersion des populations...). Cette caractéristique entraîne un coût de financement de la traction animale élevé pour le producteur.
Dans ce contexte globalement défavorable au financement de la traction animale, émergent cependant des innovations qui permettent d'espérer un développement significatif du crédit moyen terme. Le crédit bail (ou leasing) offre des perspectives satisfaisantes de sécurisation du financement de l'investissement (13). Des organisations collectives, sous forme d'association de caution mutuelle, pourraient être adaptées au financement de l'équipement agricole (11). Le partenariat entre les banques commerciales et la microfinance se renforce et offre des perspectives de mobilisation de ressource de moyen terme.

\section{CONCLUSION}

Dans l'état actuel du tissu économique et institutionnel, les perspectives de développement d'une offre de financement à grande échelle de la traction animale sont étroites dans les trois régions étudiées. Le développement de l'offre de financement de la traction animale s'insère dans la problématique plus large du financement de l'agriculture. Plusieurs voies pourraient être explorées pour renforcer cette offre. En amont, la modernisation et la sécurisation du secteur agricole familial sont des enjeux majeurs, base de toute politique de financement de l'investissement. Le renforcement institutionnel et professionnel des nouveaux acteurs que sont les organisations paysannes et les institutions de financement est un préalable au développement d'une offre financière adaptée. Des innovations techniques et organisationnelles qui permettent de sécuriser le crédit à l'agriculture et particulièrement le crédit de moyen terme demandent à être développées (fonds de garantie paritaires, associations de caution mutuelle, crédit bail, centrale de risque, mais aussi liens entre services d'appui à l'agriculture (conseil de gestion...). La sécurisation du crédit pour la traction animale sera renforcée par la reconstruction d'un système de services d'accompagnement de la traction animale. Enfin, la question de l'équipement agricole ne pourra être traitée que dans le cadre de politiques publiques de financement de l'agriculture élaborées en partenariat avec les différents acteurs du secteur agricole et en synergie avec les autres politiques économiques.

\section{BIBLIOGRAPHIE}

1. BORDET D., HAVARD M., 1998. Le financement de l'équipement agricole au Burkina Faso. Rome, Italie, FAO.

2. HAVARD M., WAMPFLER B., 2003. Les services d'appui à la traction animale au Sénégal. Rapport de recherche. In: Action thématique programmée La traction animale, composante essentielle des stratégies paysannes d'Afrique de l'Ouest et Centrale : quelles sont les pratiques de recherche à envisager face au désengagement des Etats? Montpellier, France, Cirad. (ATP n 99/70)

3. KENIKOU MOUNKANA C., 2000. Les marchés financiers ruraux. Quelles particularités pour les exploitations agricoles des provinces du Nord et de I'Extrême-Nord du Cameroun. Mémoire DEA Sciences de gestion, faculté des Sciences économiques et de Gestion, université de Ngaoundéré, Cameroun, 136 p. + annexes.

4. RAUBEC S., 2001. Le financement de la traction animale en zone de savane cotonnière du Nord-Cameroun dans un contexte de libéralisation. Mémoire Ecole supérieure d'agronomie tropicale / Cnearc, Montpellier, France, 120 p. + annexes.

5. RENARD O., 1999. Sous quelles conditions les systèmes financiers décentralisés parviennent-ils à financer l'investissement agricole ? Etude d'impact du crédit moyen terme à l'équipement de la Fececam dans le cadre de la privatisation de la filière coton au Bénin. Mémoire fin d'études Economie, Gestion, Politique économique de l'agriculture et de I'espace, Ensa, Rennes, France, 111 p. + annexes. 
6. ROESCH M., VALL E., KENIKOU MOUNKAMA C., 2002. Recettes, dépenses et crédits, comment accorder les rythmes ? Ce que peut apporter le conseil d'exploitation à la gestion de la trésorerie des exploitations agricoles et aux institutions de microfinance. In : Actes séminaire international Le financement de I'agriculture familiale dans le contexte de libéralisation : quelle contribution de la microfinance ? Dakar, Sénégal, 21-24 janv. 2002. Montpellier, France, Cirad, cédérom.

7. ROESCH M., WAMPFLER B., KENIKOU MOUNKAMA C., 2003. Financer la campagne agricole : quels appuis, quelles évolutions? Le cas du Nord Cameroun. In : Actes colloque Savanes africaines : des espaces en mutation, des acteurs face à de nouveaux défis, Garoua, Cameroun, 5-27 mai 2002. Montpellier, France, Cirad.

8. WAMPFLER B., 2001. Etude de l'offre de services financiers dans la région du Gourma (Burkina Faso). Quelles perspectives pour le financement de la traction animale ? In : Action thématique programmée La traction animale, composante essentielle des stratégies paysannes en Afrique de l'Ouest et Centrale : quelles sont les pratiques de recherche face au désengagement des Etats? Montpellier, France, Cirad-tera, 46 p. (ATP $\mathrm{n}^{\circ}$ 93-01)

9. WAMPFLER B., 2003. Coordination et pérennisation des services autour du financement de I'agriculture familiale dans la zone Office du Niger (Mali). Montpellier, France, Cirad, 66 p. + annexes. (Tera $n^{\circ} 27 / 03$ )
10. WAMPFLER B., MERCOIRET M.R., 2002. Microfinance, organisations paysannes: quel partage des rôles, quels partenariats dans un contexte de libéralisation? In: Actes séminaire international Le financement de l'agriculture familiale dans le contexte de libéralisation : quelle contribution de la microfinance ? Dakar, Sénégal, 21-24 janv. 2002. Montpellier, France, Cirad, cédérom.

11. WAMPFLER B., TRAORE Y., DIALLO A., 2002. Sécuriser le crédit à l'agriculture par des structures de cautionnement mutuel. In : Actes séminaire international Le financement de l'agriculture familiale dans le contexte de libéralisation : quelle contribution de la microfinance? Dakar, Sénégal, 21-24 janv. 2002. Fiche technique du séminaire $\mathrm{n}^{\circ} 9$. Montpellier, France, Cirad.

12. WAMPFLER B., LAPENU C., 2003. La microfinance au service de I'agriculture familiale? Paris, France, ministère des Affaires étrangères, 70 p. (Coll. Partenariats)

13. WAMPFLER B., LAPENU C., ROESCH M., eds, 2003. Actes séminaire international Le financement de I'agriculture familiale dans le contexte de libéralisation : quelle contribution de la microfinance ? Dakar Sénégal, 21-24 janv. 2002. Montpellier, France, Cirad, cédérom.

14. WAMPFLER B., 2003. Services financiers et traction animale : quelles perspectives face au désengagement de l'Etat? Synthèse des cas du Nord-Cameroun, Est Burkina, bassin arachidier du Sénégal. Montpellier France, Cirad-tera, 29 p.

\section{Summary}

Wampfler B. Financing of Draft Animal Power in the Context of State Disengagement: Learning from the Cases of NorthCameroon, Eastern Burkina Faso and the Groundnut Basin of Senegal

Accessing draft animal power with capital resources is difficult for the most vulnerable farm households because of the level of investment needed. They must then depend on loans. In West and Central Africa, public financing of draft animal power helped equip large areas where cash crop production is practiced. However, these set-ups did not prove to be sustainable and are today continuously regressing. In the vacuum left by the State, institutional innovations are developing, boosted by peasant organizations and microfinance. These two sectors are experimenting today on a very small scale with animal traction financing, but they also face major difficulties: adequate financial resources are hard to mobilize, mid-term credit is difficult to secure, and the financing supply is considerably insufficient compared to the amplitude of the demand. A few institutions, such as the CECAM network in Madagascar, for example, are experimenting innovative and promising financing modes.

Keywords: Animal power - Financing - Credit - Subsidy Farmers association - Financial policy - Agriculture - Cameroon - Burkina Faso - Senegal.

\section{Resumen}

Wampfler B. Financiamiento de la tracción animal en el contexto de desentendimiento del Estado. Aprendizajes de los casos del Norte-Camerún, del Este Burkina Faso y de la meseta de maní de Senegal

La tracción animal necesita una inversión importante que los hogares agrícolas más vulnerables pueden dificilmente realizar con fondos propios. El recurso al crédito es entonces necesario. En Africa del Oeste y central, los tipos de financiamiento públicos para la tracción animal han permitido equipar grandes zonas que practican cultivos para la renta. Pero estos dispositivos públicos son imposibles de mantener y están hoy en gran regresión. En el vacío dejado por el Estado, emergen novedades institucionales Ilevadas por las organizaciones campesinas y el microfinanciamiento. Estos dos sectores experimentan hoy, a pequeña escala, el financiamiento de la tracción animal, pero enfrentan ellos también dificultades importantes: los recursos financieros adaptados son difíciles de mobilizar, los créditos a mediano plazo son difíciles de obtener, los financiamientos ofrecidos son insuficientes con respecto a la magnitud de la demanda. Algunas instituciones, como por ejemplo la red de Cajas de ahorros y de crédito agrícola mutual (CECAM) en Madagascar, experimentan con financiamientos innovadores y prometedores.

Palabras clave: Energía animal - Financiamiento - Crédito Subsidio - Asociación de agricultores - Política financiera Agricultura - Camerún - Burkina Faso - Senegal. 